\title{
Maduración gonadal de la cucha mariposa, Glyptoperichthys gibbiceps (Kner, 1854) (Pisces: Loricariidae), en el río Guaviare, Colombia
}

\author{
Gonadal maturation the pleco butterfly Glyptoperichthys \\ gibbiceps (Kner, 1854) (Pisces: Loricariidae), \\ from the Guaviare river, Colombia
}

\section{Desenvolvimento gonadal do Glyptoperichthys gibbiceps (Kner, 1854) (Pisces: Loricariidae), do rio Guaviare, Colômbia}

\author{
Elizabeth Aya-Baquero ${ }^{1 *}$, José A. Arias-Castellanos ${ }^{2 *}$, Luis F. Collazos-Lasso ${ }^{3}$
}

1 Bióloga, MSc.

2 Biólogo, MSc, PhD

3 Ingeniero en Producción Acuícola, MSc.

* Instituto de Acuicultura de los Llanos - IALL, Facultad de Ciencias Agropecuarias y Recursos Naturales, Grupo de Investigación Chamú-Jiairé, Universidad de los Llanos, Villavicencio - Meta, Colombia.

Email: elizabeth.aya@unillanos.edu.co

Recibido: 03 de agosto de $2015 \quad$ Aceptado: 16 de agosto de 2016

\begin{abstract}
Resumen
Glyptoperichthys gibbiceps, es uno de los loricáridos de gran tamaño (hasta $50 \mathrm{~cm}$ LT), presentes en la cuenca del río Orinoco en Colombia, de la cual se sabe poco en el medio natural. Para conocer del ciclo reproductivo, fueron captura-

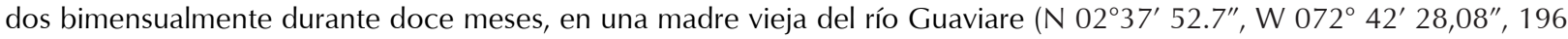
$\mathrm{msnm})$, especímenes adultos los cuales fueron sacrificados in situ, previa anestesia, registrándose las medidas morfométricas y peso, luego eviscerados, identificando el sexo y estado de desarrollo gonadal y retiradas las gónadas las cuales fueron pesadas. Una muestra de la porción media de cada gónada fue fijada en formol buferado y transferida a las 24 horas a etanol 70\% donde se conservó para procesamiento histológico estándar (H-E).

Un total de 26 hembras con peso de $495.7 \pm 52.9$ g y longitud total de $36.6 \pm 4.1 \mathrm{~cm}$ y 9 machos con peso de $676.7 \pm 192.8$ g y longitud total de $36.6 \pm 4.1 \mathrm{~cm}$, proporción sexual 3 hembras: 1 macho, fueron estudiados describiéndose la escala de maduración gonadal en cuatro estados (reposo, en maduración, maduro y un estado juvenil pre-reproductivo). En reposo los ovarios y testículos se presentan reducidos traslúcidos, de difícil diferenciación. En estado de maduración los ovarios son diferenciables ligeramente globosos y de color amarillo traslucido con ovocitos con diámetros de $2768 \pm 373 \mu \mathrm{m}$. En el estado maduro los ovarios son saculares de color amarillo intenso y brillante y los ovocitos tienen diámetros de $4155 \pm 96$ $\mu \mathrm{m}$; los testículos se presentan blancos cuando alcanzan el punto máximo de madurez. Hembras y machos adultos reproductivamente activos maduran sus gónadas una vez al año de manera sincrónica, con desove total en aguas subiendo. Se estimó una fecundidad media de $1483 \pm 380$ óvulos / hembra madura. Se describe el ciclo ovocitario y espermático de la especie como similar al de otros loricáridos.
\end{abstract}

Palabras clave: ciclo reproductivo, diámetro ovocitario, escala de maduración gonadal, maduración espermática, maduración ovocitaria 


\begin{abstract}
Glyptoperichthys gibbiceps (sailfin pleco), is one of the largest loricariids (up to $50 \mathrm{~cm}$ TL) in the Orinoco River basin in Colombia of which little is known of their natural history. To know the reproductive cycle, bimonthly collections for twelve

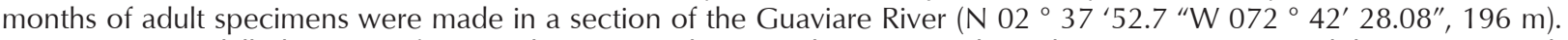
Specimens were killed on site, after anesthesia, recording morphometric and weight measurements, and then eviscerated. The gonads were weighed, and the sex and stage of sexual maturity of individual gonads were identified. A sample of the middle portion of each gonad was fixed for $24 \mathrm{~h}$ in buffered formalin and transferred to $70 \%$ ethanol until processed for standard histological analysis of tissues stained with H\&E. A total of 26 females weighing $495.7 \pm 52.9 \mathrm{~g}$ and $36.6 \pm 4.1 \mathrm{~cm}$ $\mathrm{TL}$, and 9 males of $676.7 \pm 192.8 \mathrm{~g}$ and $36.6 \pm 4.1 \mathrm{~cm} \mathrm{TL}$, (sex ratio 3 females to 1 male) were studied and four main stages were used to describe gonadal development (resting stage, developing or maturing, ripe or matured, and pre-reproductive juveniles). At rest the ovaries and testicles were small translucent and difficult to differentiate. The maturing ovaries were slightly differentiated, globular, translucent yellow containing oocytes with diameters of $2768 \pm 372 \mu \mathrm{m}$; the testicles had a rosy coloration. The mature ovaries were globular of an intense brilliant yellow, and the oocytes attained diameters of $4155 \pm 96 \mu \mathrm{m}$, and were visible to the naked eye; the testicles were white and apparent. The pre-reproductive juveniles or individuals with immature gonads were found throughout the year. In the reproductively active females and males the gonads mature synchronously once a year and spawning activity occurred during periods when water levels were rising. The average fecundity was estimated at $1483 \pm 380$ eggs / mature female. The oocyte and sperm cycles were very similar to those described for other Loricariids.
\end{abstract}

Keywords: gonadal maturation scale, oocyte diameter, oocyte maturation, reproductive cycle, sperm maturation

\title{
Resumo
}

Glyptoperichthys gibbiceps (Bode-cacunda) é um dos loricaridos de maior tamanho (até $50 \mathrm{~cm} \mathrm{CT),} \mathrm{na} \mathrm{bacia} \mathrm{do} \mathrm{rio} \mathrm{Orinoco}$ na Colômbia, de quem pouco se conhece de sua vida na natureza. Para estudar seu ciclo reprodutivo, foram capturados

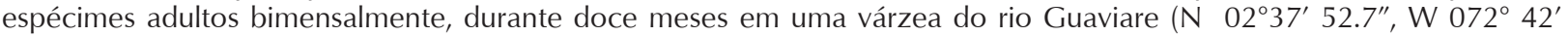
28,08", $196 \mathrm{msnm})$, os quais foram sacrificados in situ, previa anestesia, registrando suas medidas morfométricas e peso, logo foram eviscerados, identificado o sexo, estádio de crescimento gonadal e peso da gônada. Uma amostra da porção media de cada gônada foi fixada em formol tamponado e transferida às 24 horas em etanol $70 \%$, onde se conservo para processamento histológico padrão $(\mathrm{H}-\mathrm{E}$ ). Um total de 26 fêmeas (com peso de $495.7 \pm 52.9 \mathrm{~g}$ com comprimento total de $36.6 \pm 4.1 \mathrm{~cm}$ ) e 9 machos (com peso de $676.7 \pm 192.8 \mathrm{~g}$ e comprimento total de $36.6 \pm 4.1 \mathrm{~cm}$ ), com proporção sexual 3 fêmeas: 1 macho, foram estudados descrevendo sua escala de maduração gonadal em quatro estados (repouso, em maduração, maduro e um estado juvenil pre-reprodutivo). Em repouso os ovários e testículos se apresentam reduzidos translúcidos, de difícil diferenciação. Em estado em maduração os ovários são diferenciáveis ligeiramente globosos, de cor amarelo translúcido e ovócitos com diâmetros de $2768 \pm 373 \mu \mathrm{m}$; os testículos se apresentam rosáceos. No estado maduro os ovários são globosos de cor amarelo intenso e brilhante, os ovócitos conseguem diâmetros de $4155 \pm 96$ m e são visíveis ao olho nu; os testículos são brancos, diferenciáveis. Um estado juvenil pre-reprodutivo o de indivíduos com gônadas imaturas se apresentou durante o ano inteiro. Fêmeas e machos adultos reprodutivamente ativos maduram suas gônadas uma vez ao ano de maneira sincrônica, com desove total em águas subindo. Estimou-se uma fecundidade media de $1483 \pm$ 380 óvulos / fêmea madura. Descreve-se o ciclo ovocitario e espermático da espécie como similar ao de outros loricaridos.

Palavras Chave: ciclo reprodutivo, diâmetro ovocitario, escala de maduração gonadal, maduração espermática, maduração ovocitaria

\section{Introducción}

En las cuencas del Orinoco y Amazonas en Colombia, la extracción y comercialización de peces ornamentales se ha posicionado como una de las principales fuentes de ingresos para buena parte de los ribereños, actividad que genera importante impacto en los ecosistemas, por lo que se le atribuye a la captura y extracción responsabilidad significativa en la declinación que las pesquerías han registrado en los últimos años (Ortega-Lara et al., 2015).

De las 150 especies de peces de acuario que se capturan y exportan de la región de los Llanos orientales de Colombia, los loricaridos (cuchas, plecos, cascudos), han disminuido su oferta natural que fue en 2012 del
$20 \%$ del total de los peces comercializados, los que han mostrado gran descenso (AUNAP, 2013). Según Weber (2003), Glyptoperichthys gibbiceps es una de las especies con mayor afectación, debido al deterioro de los ecosistemas acuáticos por acción antrópica.

De la cucha mariposa poco se conoce de la biología en ambientes naturales. Se sabe que habita afluentes menores, caños, lagunas y madre viejas (Lasso et al., 2004; Ramírez y Ajiaco 2001), que contengan gran cantidad de materia orgánica (Landines et al., 2007), siendo ocasional encontrarle en el canal de los ríos. Es omnívora (fitoplanctófaga y detritofaga), con tendencias vegetarianas y preferencias por algas, pero incluye en su dieta invertebrados del bentos y madera 
de árboles sumergidos que soportan perifiton (Urueña et al., 2005; Landines et al., 2004). Exhibe dimorfismo sexual aparente, las hembras se diferencian de los machos porque presentan en el primer radio de la aleta pectoral asperezas más pronunciadas. Los machos maduros construyen nidos consistentes en huecos de aproximadamente $70 \mathrm{~cm}$ de profundidad y $15 \mathrm{~cm}$ de diámetro, los cuales finalizan en una bóveda más amplia, para lo anterior seleccionan las paredes de los depósitos que tengan buena sombra y allí cavan los túneles a donde conducen a las hembras para la reproducción. No se le conoce algún tipo de cortejo. En confinamiento se ha observado que varias hembras pueden poner sus óvulos en el mismo nido donde un solo macho los fertiliza y cuida al igual que las crías (Collazos-Lasso y Arias, 2009; Landines et al, 2007; Urueña et al., 2005). Cada hembra puede colocar entre 180 y 300 huevos con un diámetro de $3.53 \pm 0.35 \mathrm{~mm}$ (Urueña et al, 2005).

Considerando que los conocimientos sobre los aspectos del desarrollo gonadal de las especies se constituyen en una herramienta básica para el entendimiento y comprensión de las mismas y de sus posibilidades de producción confinada y/o conservación en la naturaleza, se realizó este estudio.

\section{Materiales y métodos}

\section{Localización}

La captura de ejemplares y sus muestreos así como el seguimientos de parámetros físico químicos del agua se realizaron durante las dos estaciones climáticas (invierno y verano), en una formación antigua del cauce del río Guaviare convertida en una madre vieja ubicada al costado izquierdo en $2^{\circ} 37^{\prime} 52.7^{\prime \prime} \mathrm{N}, 72^{\circ} 42^{\prime} 28,08^{\prime \prime}$ W y 196 msnm. Es un cuerpo de agua rodeado de un bosque de galería denso que deposita gran cantidad de ramas, troncos y hojarasca en el cuerpo de agua, con un fondo cubierto de una capa de lodo y materia orgánica de $20 \mathrm{~cm}$ en promedio de profundidad, lo que determina un agua ácida de color té.

\section{Peces de estudio}

Bimensualmente (febrero 2013 - abril 2014), se realizó trabajo de campo consistente en toma y análisis de muestras de agua (Boyd 1997), y colecta de peces mediante uso de mallas de nylon de $6 \mathrm{~cm}$ de ojo de maIla, las cuales eran colocadas a lo ancho de la madre vieja y luego los ejemplares eran asustados mediante golpes sobre las palizadas para que en su huida quedaran atrapados en la malla. Los especímenes captu- rados mayores de $30 \mathrm{~cm}$ de longitud estándar, fueron medidos (longitud total y longitud estándar), y pesados in situ, y luego anestesiados (Metanosulfonato de Tricaina, MS-222 ${ }^{\circledR}, 200$ ppm), hasta cuando disminuyeron los movimientos operculares y no respondieron a estímulos externos (Johnson et al., 2009), luego fueron sacrificados mediante corte medular. Enseguida cada ejemplar fue disectado, removido el intestino y vísceras y determinado el sexo y estado de desarrollo gonadal, para esto último se consideró el color, aspecto, tamaño y disposición de las gónadas en la cavidad abdominal. Para los ovarios, además, se registró el peso, la visualización o no de ovocitos y cuando fueron visibles, el color y homogeneidad de los mismos.

\section{Determinación del desarrollo ovocitario}

Para la determinación del crecimiento de los ovocitos se procedió como sigue: una vez definido el estado del desarrollo de los ovarios se extraían, se medían (largo y ancho del ovario), pesaban en balanza de precisión 0,001 g y una porción de la región media de cada ovario equivalente a 0,01 g era fijada en solución de formol buferado al $10 \%$ por 24 horas al cabo de las cuales la muestra era transferida a alcohol del $70 \%$ y conservada para realizar los cortes histológicos que fueron los de rutina con Hematoxilina-Eosina $(\mathrm{H}$ y E), (Johnson et al., 2009; Arias et al., 2004; Vazzoler, 1996).

Tres sub-muestras de un gramo c/u de los remanentes de los ovarios fueron fijadas y mantenidas en formol al $4 \%$ y en el Laboratorio del Ictiología del Programa de Biología de la Universidad de los Llanos, a cada una se le contó el número de ovocitos para el propósito de determinar la fecundidad absoluta. Una sub-muestra conservada se colocó en solución de $\mathrm{NaCl}$ al $0.9 \%$ e inmediatamente los ovocitos fueron disgregados y sus diámetros medidos utilizando estereoscopio Nikon convencional con reglilla en el ocular calibrada previamente con cámara de Neubauer.

Para el estudio microscópico de los testículos se procedió similar a las hembras: una vez definido el estado del desarrollo de los testículos se extrajeron, se midieron (largo en $\mathrm{cm}$ ), pesaron en balanza de precisión 0,001 g y una porción de la región media de cada testículo fue fijada.

La identificación histológica de las diferentes fases de crecimiento de las células germinales se realizó utilizando microscopio con cámara fotográfica Carl Zeiss.

La Índice Gonadosomático (IGS) fue calculada mediante la fórmula: 


$$
\mathrm{IGS}=\mathrm{PG} / \mathrm{PT} \text { * } 100
$$

Dónde:

$\mathrm{PG}=$ peso de la gónada $\mathrm{y}$

PT = peso total (Liang y Shieh, 2005).

\section{Resultados}

Fueron capturados un total de 77 ejemplares, 42 de ellos fueron registrados, medidos, pesados y luego liberados por ser de talla menor a $30 \mathrm{~cm}$. Treinta y cinco fueron sacrificados 26 fueron hembras (74.28\%) que registraron un peso de $495.7 \pm 52.9 \mathrm{~g}$ y una Longitud Total (LT) de $36.6 \pm 4.1 \mathrm{~cm}$, y 9 fueron machos $(25.71 \%)$ con $676.7 \pm 192.8 \mathrm{~g}$ de peso y $41.3 \pm 2,6 \mathrm{~cm}$ de LT, significativamente mayores los machos, con una relación 3 hembras: 1 macho.

Las hembras poseen dos ovarios globulares y saculares, asimétricos cuando maduros, siendo el ovario izquierdo un 20 a $30 \%$ más grande (figura 1). Son órganos libres ventro-caudales que se unen en un oviducto corto que termina en un poro genital detrás del ano. Varían en volumen y color a lo largo del ciclo de desarrollo, desde alargados y traslúcidos al inicio de la maduración y hasta globosos y amarillo intenso cuando maduros.

Los preparados histológicos mostraron cinco fases de crecimiento ovocitario que se pueden asociar en dos grupos: uno de crecimiento pre-vitelogénico donde aumenta el tamaño nuclear, el número de nucléolos y al final se insinúan las membranas foliculares. Los ovocitos pre-vitelogénicos se encuentran presentes duran-

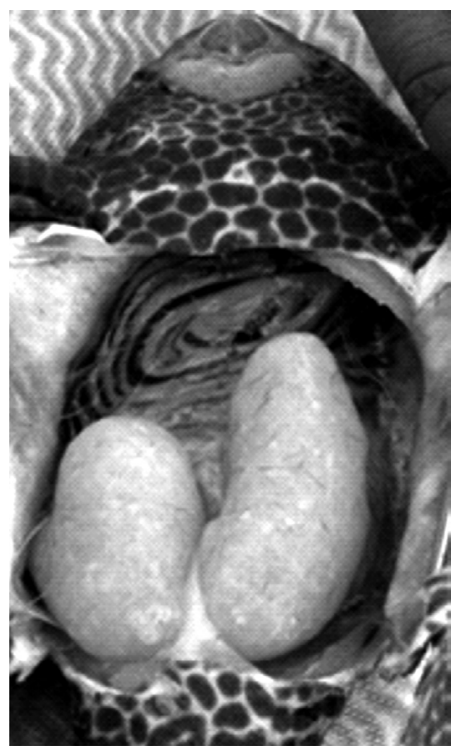

Figura 1. Ovarios maduros en la cavidad abdominal de G. gibbiceps. te todo el ciclo anual del desarrollo sexual y se pueden distinguir los más pequeños como ovocitos cromatina nucléolo, escasos y difíciles de señalar, y ovocitos perinucleolares, los más abundantes en todo el estudio y estados de desarrollo del ovario. El segundo grupo de ovocitos estuvo constituido por dos tipos celulares vitelogénicos, el grupo de menor diámetro presentó ovocitos menores de $1500 \mu \mathrm{m}$ que se diferenciaron por poseer alvéolos corticales, y los ovocitos vitelogénicos propiamente, que son células grandes conteniendo gran cantidad de vitelo y encerrados por las envolturas foliculares.

\section{Escala de Maduración}

La escala de maduración gonadal de hembras puede establecerse como sigue (adaptado de Vazzoler, 1996):

\section{Estado I - En reposo}

En la localidad colectada, éste estado se presentó generalizado en las hembras entre mediados de junio hasta mediados de noviembre. Los ovarios fueron pequeños $(2.9 \pm 0.6 \mathrm{~g})$. Fueron estructuras finas traslúcidas de difícil diferenciación. Los cortes histológicos mostraron ovocitos peri-nucleolares predominantemente de tamaño pequeño (menores de 300 m de diámetro).

\section{Estado II - En maduración}

Los ovarios presentaron forma alargada y ligeramente globosa, ocupando $1 / 3$ de la cavidad abdominal, el color fue amarillo pálido, con irrigación sanguínea notoria, se presentaron frecuentemente entre noviembre a febrero, el peso fue de $18.7 \pm 1.7$ g. Histológicamente se pudo reconocer dos tipos de células predominando, unas de menor tamaño de tipo peri-nucleolar grandes (500 $\mu \mathrm{m}$ de diámetro) y otras de tamaño similar con alvéolos corticales (figura 2).

\section{Estado III - Maduro}

Hacía el final del periodo seco, en el mes de febrero, los ovarios empiezan a experimentar un crecimiento rápido incrementando su peso y el diámetro de los ovocitos como resultado del depósito de vitelo. Para el periodo de febrero a marzo-abril los ovarios pasan a ser totalmente de forma sacular, ocupan hasta $2 / 3$ de la cavidad abdominal y su color se vuelve amarillo intenso, adquiriendo un peso de $48.3 \pm 9 \mathrm{~g}$. Los cortes histológicos caracterizan macro-células envueltas en membranas conformando folículos con ovocitos ma- 


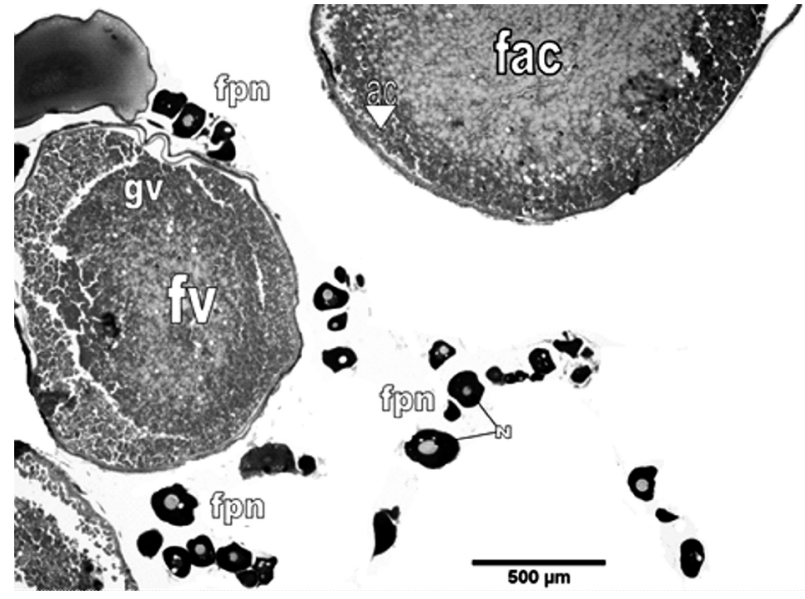

Figura 2. Fotomicrografía de ovocitos en diferentes fases de maduración de Glyptoperichthys gibbiceps. Fase perinucleolar (FPN), fase de alvéolo cortical (fac), fase de vitelogénesis (fv). Núcleo (N), alvéolo cortical (ac), granulo de vitelo (gv). HE. 10X.

duros que serán expulsados al momento del aumento de las lluvias (figura 3). El conteo de ovocitos maduros permitió calcular una fecundidad media de $1483 \pm$ 380 ovocitos / hembra madura.

Algunos ejemplares colectados en el mes de abril mostraron ovarios relajados rosados translúcidos que pesaron $6.6 \pm 2.8 \mathrm{~g}$. En el estudio histológico se observaron cicatrices de diferente tamaño invadidas por células fagocitarias. Para algunos autores se trata de un estado de pos-desove, que dura muy poco tiempo, no más de 10 días.

\section{Estado IV - Inmaduro}

Doce hembras sacrificadas mayores de $30 \mathrm{~cm}$ de LT, fueron ejemplares inmaduros, esto es con ovarios alargados, incoloros y transparentes, difíciles de dife-

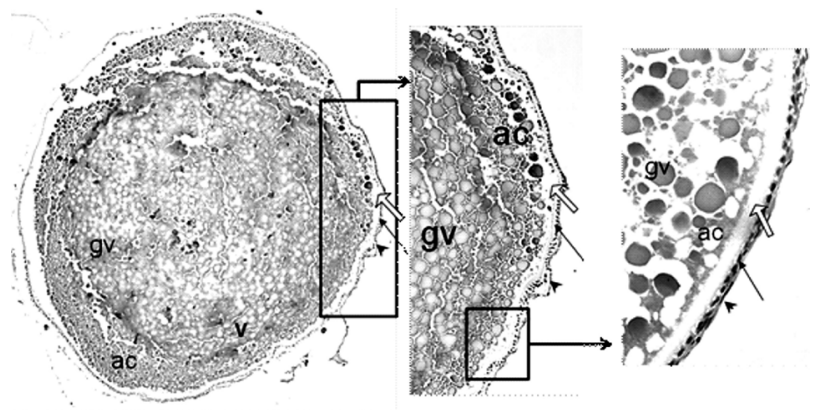

Figura 3. Fotomicrografía de ovocito en fase de maduración de Glyptoperichthys gibbiceps. Izquierda 4X. Centro 10X. Derecha 40X. Grano de vitelo (gv), alveolo cortical (ac). Membranas foliculares, teca (punta de flecha), granulosa (flecha), radiada (flecha blanca). $\mathrm{HE}$. renciar. Los cortes histológicos apenas mostraron una especie de estructura de tejido conectivo sin precisarse cuales de las células inmersas en él pudieran ser ovogonias. Los ovarios en estado de reposo son presentaron pesos de $2.93 \pm 0.6 \mathrm{~g}$, ocupando menos del $10 \%$ de la cavidad celómica, de color rosado pálido.

Los testículos fueron simétricos, alargados y ubicados dorso-lateralmente en la cavidad abdominal. Son de volumen reducido y color variable según el estado de desarrollo que presenten, siendo que son digitiformes como en otros silúridos neo tropicales, desde traslúcidos hasta semi-globosos y blancos cuando maduros.

Los testículos contienen túbulos seminíferos profusos y apilados sobre sí mismos. Cada túbulo a su vez soporta las células germinativas en grupos protegidos por las células císticas que forman múltiples cistos (espermatocitos, espermátides y espermatozoides).

Las fases de desarrollo de las células germinativas en los machos que pudieron ser reconocidas fueron:

Espermatocitos. Las células de mayor tamaño observadas. Contienen un núcleo grande (al menos la mitad del tamaño celular), de citoplasma escaso y periferia irregular. Los cistos que los contienen son los más grandes en el túbulo seminífero (Figura 4). No fue posible visualizar los dos tipos de espermatocitos que se reportan de manera normal para teleósteos.

Espermátides. Fueron células más pequeñas que los espermatocitos pero más abundantes en los cistos. El desarrollo de ellas (espermiogénesis), conduce de una célula redondeada de núcleo grande a una con un núcleo y citoplasma reducido que deja ver el esbozo del flagelo.

Espermatozoides. Fueron las células germinativas más pequeñas en el centro de los cistos. Son flageladas muy coloreadas y con poco citoplasma, abundan también en la luz del túbulo. (Figura 4).

\section{Escala de maduración testicular}

Estadio Inmaduro. Los testículos se presentaron como órganos muy finos, transparentes y con apariencia de hilos sobre la superficie ventral de la vejiga natatoria. Difíciles de diferenciar, solo dos ejemplares los mostraron. Histológicamente los túbulos seminíferos fueron pequeños (15 $\mu \mathrm{m}$ de diámetro o menos), mal delimitados, sin luz y sin cistos evidentes. Se presentaron tapizados internamente por células epiteliales difíciles de diferenciar de las espermatogonias. 


\section{Estado I de reposo}

Es el estado de mayor duración, se presentó entre mayo y febrero. Los testículos apenas si se pueden diferenciar, son filiformes y transparentes. Los cortes histológicos dejan ver túbulos seminíferos desordenados, compactos y rellenos por tejido conectivo laxo sin evidencia de espermatogonias.

\section{Estado II, En maduración}

Es un estado relativamente corto en la especie (enero a mayo), durante este tiempo los testículos aumentaron de tamaño de manera permanente, siendo que la región caudal crece vertiginosamente solo al final del estado al tiempo que se ramifica. La coloración varió desde transparente blanquecina hasta blanca crema. Aparentemente crecen lentamente entre enero y febrero predominando túbulos con cistos conteniendo espermatocitos (figura 4).

Pero para febrero - abril los túbulos a más de aumentar su diámetro (hasta $100 \mu \mathrm{m}$ ) y luz, los cistos solo tienen espermátides y espermatozoides dentro de ellos.

\section{Estado III, Maduro}

Los testículos en abril presentaron su máximo tamaño y son claramente digitiformes de color blanco, túrgidos y ocupan al menos el $15 \%$ de la cavidad celomica con peso de hasta 0,4 g. Histológicamente los túbulos presentaron también su máximo diámetro (más de $150 \mu \mathrm{m}$ ). El lumen de cada túbulo se llena de espermatozoides libres en líquido seminal.

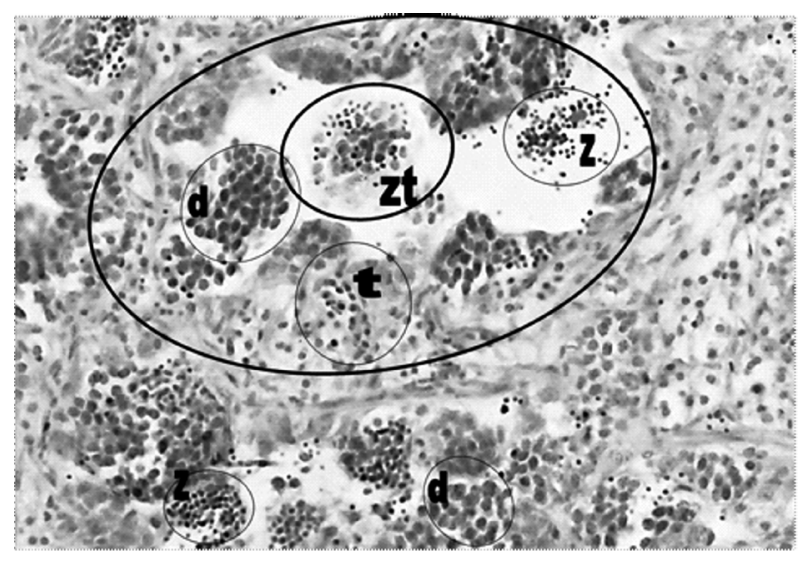

Figura 4. Fotomicrografía de corte transversal de testículo de Glyptoperichthys gibbiceps, en estado de maduración. Circulo externo límite del túbulo seminífero, circulo interno contorno de la luz del túbulo. Cisto de espermatocitos (t), cisto de de espermátides (d), cisto de espermatozoides (z), espermatozoides en la luz del túbulo (zt). ). HE. Objetivo: 40X.

\section{Estado IV En reposo}

Testículos rojizo translucidos y flácidos, apenas distinguibles.

Los valores de IGS más altos (9.7 \pm 0.8$)$ para hembras se presentaron durante el periodo de lluvias altas (marzo y abril), que coincide con la época de reproducción, y de (3.5 \pm 0.6$)$ en enero y febrero cuando se da inicio al depósito masivo de vitelo, muy similar a lo descrito por Hernández, 2008, para Pterigoplichthys pardalis y Vinicius et al, 2002 para Loricariichthys platymetopon.

Las hembras maduras presentaron una fecundidad absoluta de $1418 \pm 360$ ovocitos, con diámetros de $4048 \pm 0.24 \mu \mathrm{m}$ durante la temporada de lluvias altas previamente al desove (aguas subiendo). Los ovocitos fueron de color amarillo brillante intensificándose a medida que aumenta la maduración y se acerca al desove.

\section{Discusión}

La morfología en la maduración del ovario y del testículo de cucha mariposa fue semejante a la descrita para otros loricaridos tropicales como Farlowella vittata por García-Alzate et al., 2012, Hypostomus affinis por Duarte et al., 2011 y Mazzoni y Caramaschi, 1997; Hypostomus commersonii y Plecostomus commersonii por Agostinho et al., 1990, 1982 respectivamente; Hypostomus ternetzi, Megalancistrus aculeatus por Suzuki et al., 2000, Pterigoplychthys pardalis por WakidaKusunoki et al., 2011. La escala se inició con ovarios pequeños (estado de reposo), sé continuó con un periodo en el cual los ovarios crecen permanentemente y acumulan vitelo (estado de maduración) y concluye con un estadio de completo desarrollo (estado maduro) (Arias 2002). La ovogénesis se asemeja a lo que ocurre en otros peces teleósteos y se adelanta en dos grandes etapas. La primera de crecimiento primario agrupa los ovocitos del lote de reserva. La segunda de crecimiento secundario agrupa los ovocitos en maduración (Arias et al., 2004; Vazzoler, 1996; Guraya, 1994, Wallace y Selman, 1981).

En la etapa inicial del desarrollo de los ovocitos la fase de cromatina nucléolo observada fue semejante a la descrita por Arias et al., 2004 y se presentó hacia la mitad del estado de reposo. La fase peri-nucleolar fue visible durante todo el ciclo reproductivo siendo la célula característica de crecimiento primario pre-vitelogénico (Junquera, 1996). Cuando la fase peri-nucleolar se aproxima a su final (cerca de las $500 \mu \mathrm{m}$ de diámetro), se logran visualizar las tres membranas foliculares (Agostinho et al., 1990, 1982), anunciando así dos su- 
cesos fundamentales: el primero de reclutamiento y el segundo de inicio de la etapa de depósito de reservas, los ovocitos perinucleolares reclutados para continuar el desarrollo se crecen rápidamente a células de alvéolos corticales periféricos que depositan los factores relacionados con los procesos de fertilización (Carrillo y Zanuy, 1993). El rápido crecimiento de los ovocitos en la fase vitelogénica (en tan solo dos meses como máximo), se debió al depósito de vitelo como lo anotaron Wallace y Selman, 1981. Los ovocitos maduros fueron las células más grandes del ciclo de maduración.

Como ocurre en muchas otras especies de peces nativos en el ovario de cucha mariposa coexisten dos lotes diferentes de ovocitos, uno en crecimiento primario (lote de reserva) y otro en crecimiento secundario (lote de desove), lo que lo clasifica como un ovario de desarrollo sincrónico por grupos con desove total (Vazzoler, 1996; Zanuy y Carrillo 1995; Wallace y Selman, 1981).

El periodo de reproducción ha sido comúnmente determinado para especies de loricaridos a través del cálculo del valor de la relación gónado-somática (García-Alzate et al., 2012). Las mayores IGS coincidieron con la mayor frecuencia del estadio maduro para los meses de marzo y abril y el aumento de las lluvias, semejante a lo reportado por Wakida-Kusunoki y Amador, 2011; Liang et al, 2005; Arias et al., 2004; Suzuki et al., 2000; Lowell Mc Connell 1999; Vazzoler 1996.

Los testículos de cucha mariposa fueron similares en su morfología general y maduración al de otros silúridos tales como Pseudoplatystoma corruscans por Lopes et al., 1987; Rhinelepis aspera por Agostinho et al., 1987 y Plecostomus albopunctatus por Antoniutii et al., 1985.

En el testículo tubular se desarrollaron los diferentes tipos de células de manera continua en los cistos muy semejante a los descrito para otras especies de peces neotropicales como Brycon amazonicus por Arias, 2002; Brycon orbignyanus por Zaiden, 1997.

No es posible inferir sobre las espermatogonias pues no fueron visualizadas, pero las fase de espermatocito fue semejantes a las descritas para Rhinelepis aspera por Agostinho et al., 1987. La espermiogénesis ocurrió dentro de los cistos sin poderse evidenciar los movimientos propios de esta fase como lo describen Carrillo y Zanuy, 1993. Los espermatozoides son similares a los de otros teleósteos, dentro de los cistos y libres en la luz del túbulo, como lo reportaron Arias, 2002; Zaiden, 2000. La escala de maduración testicular en cuatro estadios básicos que se propone para el ciclo anual de machos adultos siguiendo los delineamientos del trabajo clásico de Barbieri et al., 1981.

\section{Conclusiones}

El ovario de cucha mariposa fue un órgano en saco (cistovario), con desarrollo de los ovocitos de manera sincrónica por grupos y con desove total. El ciclo anual es de cinco fases de desarrollo de los ovocitos en dos etapas, la primera de crecimiento pre-vitelogénico contiene ovocitos que perduran durante todo el ciclo, los ovocitos perinucleolares. La segunda etapa es la vitelogénesis propiamente que se efectúa mediante aumento de las envolturas foliculares y del tamaño celular de la ovocélula por depósito de vitelo.

Los testículos fueron órganos pares como los de muchos otros peces teleósteos, con forma y funcionamiento tubular. La espermatogénesis se efectúa en cistos en los cuales sucesivamente aparecen 4 fases: de espermatogonia (no diferenciada en este trabajo), espermatocito, espermátide y espermatozoide. Cada túbulo seminífero enrollado sobre si mismos contiene cientos de cistos y cada cisto células germinativas en una misma fase de desarrollo.

La escala de maduración de la especie se pudo definir en cuatro estados: inmaduro, maduración, maduro y reposo. El mayor valor de la relación gonadosomática coincidió con la presencia de las fases y estados maduro en ambos sexos durante la época de lluvias (aguas subiendo), en los meses de marzo y abril.

\section{Referencias}

Agostinho AA, Barbieri MC, Agostinho CS, Barbieri C. Biologia reprodutiva de Rhinelepis aspera (Agazzis, 1829) (TELEOSTEI: LORICARIIDAE), no rio Paranapanema. I. Estrutura dos testículos e escala de maduridade. Rev Bras Biol. 1987;47(3):309-317.

Agostinho AA, Segatti HN, Agostinho CS. Ciclo reproductivo e primera maturação de fêmeas de Hypostomus commersonii (Valenciennes, 1840) (Siluriformes Loricariidae), no reservatório Capivari-cachoeira, PR. Rev Bras Biol. 1990;51(1): 31-37.

A gostinho AA, Narahara MY, Godinho HM. Morfologia dos ovários de Plecostomus commersonii (Valenciennes, 1840), (Osteichthyes Loricariidae): Desenvolvimento dos ovocitos e escala de maturidade. Rev Bras Biol. 1982;42(1):71-77.

Antoniutti DM, Godinho HM, Ranzani-Paiva MJT. Estrutura da população, reprodução e crescimento do cascudo Plecostomus albopunctatus, Regan, 1908. (Ostheichthyes, Loricariidae) do Rio Jaguarí, São Paulo. Brasil. Boletim do Instituto de Pesca. 1985;12:121-131.

Arias CJA, Zaniboni-Filho E, Pardo-Carrasco SC, Vásquez-Torres W. Ovogénesis de yamú Brycon siebenthalae (Teleostei: Characidae), en Cautiverio. Actual Biol. 2004;26(81):171-183. 
Arias-Castellanos JA. 2002. Biología reproductiva del yamú Brycon siebenthalae (Pisces: Characidae), en cautiverio. Tesis Doctoral. Universidad del Valle, Cali. 116 p.

AUNAP - Autoridad Nacional de Acuicultura y Pesca. 2013. Diagnóstico del estado de la acuicultura en Colombia. Bogotá, Colombia. 44p.

Barbieri MC, Barbieri G, Marins MA. Sobre a anatomiae histologia de testículo de Geophagus brasiliensis (Quoy y Gaimard, 1824) na Represa do Lobo, Estado de São Paulo.Rev Brasil Biol. 1981;41(1):169-173

Boyd CE. Practical aspects of chemistry in pond aquaculture. Progressive Fish-Culturist. 1997;59:85-93.

Carrillo M, Zanuy S. 1993. Fisiología de la Reproducción de los Teleósteos. En: Castelló OF (ed.). Acuicultura Marina, Fundamentos biológicos y Tecnologia de la producción. Universidad de Barcelona, España, 123-166.

Collazos-Lasso LF, Arias CJA. Estimulación a la maduración final y el desove de Ancystrus triradiatus. Orinoquia. 2009;13(1):14-19.

Duarte S, Araújo FG, Bazzoli N. Reproductive plasticity of Hypostomus affinis (Siluriformes: Loricariidae) as a mechanism to adapt to a reservoir with poor habitat complexity. Zoologia. 2011;28(5):577-586.

García-Alzate CA, Román-Valencia C, Barrero AM. Biología alimentaria y reproductiva de Farlowella vittata (Siluriformes: Loricariidae), en la cuenca del río Güejar, Orinoquía, Colombia. Rev Biol Trop. 2012;60(4):1873-1888

Guraya SS. Gonadal Development and Production of Gametes in Fish. Proc Indian Nat Sci Acad B. 1994;60(1):15-32.

Hernández SM. 2008. Aspectos reproductivos del loricarido Pterigoplichthys pardalis (Castelnau, 1855), en la laguna de las Ilusiones. Tesis de grado. Tabasco. México. 34-40. Pp.

Johnson R, Wolf J, Braunbeck T. 2009. OECD Guidance Document for the Diagnosis of Endocrine-Related Histopathology of Fish Gonads. Draft of January 7.

Landines MA, Sanabria OAI, Daza PV. 2007. Producción de peces ornamentales en Colombia. INCODER. Bogotá, Colombia. pp. $23-27$.

Landines P MA. Urueña B FR. Ávila T E. Rodríguez VL. 2004. Guía de producción de peces ornamentales "Loricaridos" de la Orinoquía Colombiana. Ministerio de Agricultura y Desarrollo Rural. Universidad Nacional de Colombia. Sede Bogotá.

Lasso C, Mojica JI, Usma JS, Maldonado J, Donascimiento C, Taphorn D, et al. Peces de la Cuenca del Río Orinoco. Parte I: Lista de especies y distribución por subcuencas. Biota Colombiana. 2004;5(2):95-158.
Liang SH, Shieh B. Size structure, reproductive phenology, and sex ratio of an exotic armored catfish (Liposarcus multiradiatus), in Kaoping River of Southern Taiwan. Zoological Studies. 2005;44(2):252-259.

Lopes RA, Lopes OV, Paula CV, Dos Santos HSL, Sobrinho A. On the reproduction of brazilian fishes, IX. Spermatogenesis of the surubim Pseudoplatystoma corruscans Agassiz, 1829 (PISCES: PIMELODIIDAE). Ars Vet. 1987;3(1):17-22.

Mazzoni R, Caramaschi EP. Spawing season, ovarian development and fecundity of Hypostomus affinis (Osteichthyes, Loricariidae). Rev Bras Biol. 1997;57(3):455-462.

Ortega-Lara AY, Cruz-Quintana V, Puentes A. 2015. Dinámica de la actividad pesquera de Peces Ornamentales Continentales en Colombia. Serie Recursos Pesqueros de Colombia - AUNAP. Fundación FUNINDES. 174p.

Ramírez GRH, Ajiaco RE. 2001. La pesca en la baja Orinoquia Colombiana: una visión integral. Bogotá: MADR/PRONATA/COLCIENCIAS/INPA. Produmedios. 199-202 Pp.

Suzuki HI, Agostinho AA, Winemiller KO. Relationship between oocyte morphology and reproductive strategy in loricariid cat Fishes of the Paraná River, Brazil. J Fish Biol. 2000;57:791-807.

Urueña F, Ávila E, Rodríguez L, Landines MA. 2005. Guías de producción de peces ornamentales de la Orinoquía colombiana. Loricaridos. Universidad Nacional de Colombia. MVZ. Bogotá. $85 \mathrm{p}$.

Vazzoler AE. 1996. Biología de reprodução de peixes teleósteos: teoría y prática. Edit. Nupelia, Maringá- R.169 p.

Vinicius MQM, Querol E, Gomes NNA. Fator de condição gonadal, índice hepatossomático e recrutamento como indicadores do período de reprodução de Loricariichthys platymetopon (Loricariidae), bacia do rio Uruguay médio, sul do Brasil. Iheringia, Sér. Zool. Porto Alegre. 2002;92(3):79-84.

Wakida-Kusunoki AT, Amador-del Ángel LE. Aspectos biológicos del pleco invasor Pterygoplichthys pardalis (Teleostei: Loricariidae) en el río Palizada, Campeche, México. Rev Mex Biodiv. 2011;82(3):870-878.

Wallace RA, Selman K. Celullar and dynamic aspects of oocyte growth in teleosts. Amer Zool. 1981;21:325-343.

Weber C. 2003. Subfamily Hypostominae (armored catfishes). In Check list of the freshwater fishes of south and central America, R. E. Reis, S. O. Kullander y C. J. Ferraris, Jr. (eds.). EDIPUCRS, Porto Alegre, Rio Grande do Sul. 729 Pp.

Zaiden FS. 1997. Estructura testicular da piracanjuba Brycon orbignianus (Valenciennes, 1849) (PISCES: CHARACIDAE), nos vários estádios do ciclo sexual. Dissertação de Mestrado. CAUNESP-UNESP, Jaboticabal, Brasil. 125p. 